\title{
Useful visual field size for pattern perception
}

\author{
SHINYA SAIDA and MITSUO IKEDA \\ Tokyo Institute of Technology, Yokohama 227, Japan
}

\begin{abstract}
The useful visual field size at each fixation in a pattern was investigated by artificially supplying various visual field sizes on a TV display. The degree of pattern perception was measured in terms of recognition memory for pictures, and the speed of processing pictures was determined as a function of field size. A serious deterioration in the perception of pictures occurred as the visual field was limited to a small area around the fovea (about $3.3^{\circ} \times 3.3^{\circ}$ ), processing speed becoming extremely slow. Speed increased gradually as visual field size became larger, to reach a certain level beyond which no further increase was observed. The visual field size at this asymptotic speed was called the useful visual field and was found to be about $50 \%$ of the entire pattern size. Analysis of eye-movement records demonstrated that in terms of the useful visual field, the scanning characteristics of the eye over the pattern occurred in a heavily overlapping manner to assure good perception of the pattern.
\end{abstract}

When a person looks at a picture, the eyes remain fixed between saccades, the fixation pauses lasting about $.3 \mathrm{sec}$ each and the eyes being fixated about $90 \%$ of the time. During the other $10 \%$ of the time, the eyes are moving from one fixation point to another at very high speed. Virtually all information is acquired during the fixation pauses, because the ability to acquire visual information decreases during saccadic movements, as shown by various authors (Latour, 1962; Volkmann, 1962; Volkmann, Schick, \& Riggs, 1968; Zuber \& Stark, 1966).

A major experimental question is how much of the field of view is needed at each fixation pause to recognize a picture. We define the useful field of view as the area around the fixation point from which usable information for the recognition of the whole picture is extracted. There have been several works on determination of the size of this field of view for a single fixation (Antes \& Edwards, 1973; Chaikin, Corbin, \& Volkmann, 1962; Edwards \& Goolkasian, 1974; Engel, 1971; Ikeda \& Takeuchi, 1975; Sanders, 1970). Determination of the useful visual field for free eye movement over the pattern requires a special experimental arrangement. It would seem reasonable to assume that the size of the useful visual field is equal to the size of saccadic movements. In this way, the picture would be covered by the useful visual field most efficiently, that is, without any redundancy. We have shown previously, however, by artificially restricting the visual field, that this assumption is incorrect in the case of reading sentences and that the span of apprehension is wider than the size of the saccade (Ikeda \& Saida, 1978). Results by McConkie and Rayner (1975) suggest a similar conclusion.

The present address of Shinya Saida, from whom reprints ma) be obtained: Industrial Products Research Institute, 21-2. 4-chome Shimomaruko Ota-ku, Tokyo 146, Japan.
In previous experiments, the size of the visual field was artificially restricted by covering the pattern with a piece of cardboard out of which was cut a viewing hole (Becker, 1935; Hochberg, 1968; Yamane, 1935). The subject could see the whole pattern only by moving the cardboard with his hands. This enabled investigation of the role of eccentric vision. In more recent experiments, the subject's own eye movements have been used. Watanabe (1971) used a TV-display system to examine pattern recognition ability with a visual field size of $3^{\circ}$ of visual angle and showed that pattern recognition became very difficult at that size. Andreeva, Verghiles, and Lomov (1972) used a special suction cup fixed on the eye to limit the visual field size to $3^{\circ}$. Their subjects showed extreme difficulty in performing visual problem solving tasks. Andreeva et al. did not alter the visual field size systematically, however, and were unable to derive a critical visual field size at which such difficulty begins.

The aim of the present experiment was, therefore, to investigate how far into the periphery visual information is utilized and to determine quantitatively the size of the useful visual field when subjects observe ordinary pictures. A special apparatus by which the visual field size could be freely altered was employed. The speed of processing pictures was obtained as a function of the visual field size. The investigation consisted of a large-picture experiment and a smallpicture experiment. The main difference between experiments was the size of pattern presented. Different sizes were employed to investigate the influence of the pattern size upon the size of the useful visual field.

\section{GENERAL METHOD}

\section{Apparatus}

The apparatus was similar to that used previously (Ikeda \& 


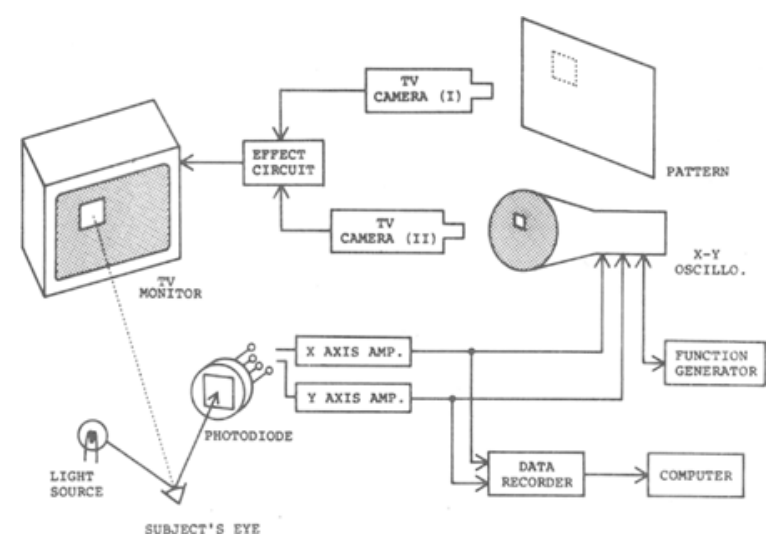

Figure 1. Schematic view of the appanatus to restrict visual field size.

Saida, 1978; Ikeda, Saida, \& Sugiyama, 1977). The scheme of the experimental arrangement is shown in Figure 1. A subject viewed a television monitor with the right eye from a distance of $1 \mathrm{~m}$, the left eye being covered completely. The picture on the TV monitor appeared to the subject only partly within a small square whose position coincided exactly with his visual axis. Whenever eye position changed, a different part of the picture, corresponding to the area surrounding the subject's new fixation point, was shown. In order to realize this condition, the twodimensional eye movements of the subject's right eye were detected by the corneal reflection method, using a Schottoky barrier silicon photodiode placed in front of the subject's eye. The outputs of the photodiode were fed to an X-Y oscilloscope so as to control the horizontal and vertical position of the bright square that was generated on the oscilloscope by the function generator. This bright square was used as a gate signal in an effect circuit by inputting it through Camera II.

A stimulus picture was fed into the TV monitor through Camera I via the effect circuit. Only that portion of the pattern which corresponded to the position and the size of the bright square on the oscilloscope appeared on the TV monitor screen.

A preset digital timer controlled the duration of picture presentation on the TV screen. The luminance of the pictures on the TV monitor was $.2 \mathrm{~cd} / \mathrm{m}^{2}$ in the black part, and $6.5 \mathrm{~cd} / \mathrm{m}^{2}$ in the white part. The subjects used a bite board to immobilize their heads.

\section{Stimuli}

The stimuli were 1,600 drawings taken from illustrations in novels, newspapers, and sketchbooks. Subject matter covered a wide range, including Japanese family scenes-31\%, landscapes$24 \%$, foreign family scenes-10\%, sculptures-7\%, histology scenes $-4 \%$, and single objects (vehicles, animals, flowers, foods) $-24 \%$. Pictures that had distinctive features that were easy to remember were eliminated and those which were too similar to others in content were avoided. These drawings might be characterized as a highly variegated collection of heterogeneous patterns.

The pictures were presented in two different sizes on the TV monitor screen: $14.4^{\circ} \times 18.8^{\circ}$ (large-picture experiment) and $10.2^{\circ} \times 13.3^{\circ}$ (small-picture experiment). The latter size was $50 \%$ of the area occupied by the former picture. Two examples of the pictures are shown at the top of Figure 2.

\section{Subjects}

The subjects were nine male undergraduate and graduate students, ranging in age between 20 and 27 years. Five of the subjects participated in the large-picture experiment, and the other four subjects participated in the small-picture experiment. Four subjects wore glasses, but the apparatus was able to detect the corneal reflection image (the first Purkinje image) through glasses without biasing the eye-movement recordings.

\section{LARGE-PICTURE EXPERIMENT}

\section{Procedure}

The speed of processing pictures was defined as the reciprocal of the exposure duration of pictures to yield $70 \%$ pattern recognition. To do this, the duration vs. degree of pattern-recognition curves were determined according to Potter and Levy (1969) for various field sizes by pattern-recognition memory tests consisting of a study phase and a test phase. The durations at the $70 \%$ point were read from the curves, which gave a curve relating the speed of processing pictures to field size.

Five different visual field sizes were employed: $3.3^{\circ} \times 3.3^{\circ}$, $4.6^{\circ} \times 4.6^{\circ}, 7.3^{\circ} \times 7.3^{\circ}, 11.4^{\circ} \times 11.4^{\circ}$, and nonrestricted size. The firsi four covered $4 \%, 8 \%, 19 \%$, and $48 \%$ of the area of the entire picture $\left(14.4^{\circ} \times 18.8^{\circ}\right)$. For each visual field size, four exposure durations were employed: $5,7,10$, and $20 \mathrm{sec}$ for the $3.3^{\circ} \times 3.3^{\circ}$ visual field; $3,5,10$, and $20 \mathrm{sec}$ for the $4.6^{\circ} \times 4.6^{\circ}$ visual field; $1,3,10$, and $20 \mathrm{sec}$ for the $7.3^{\circ} \times 7.3^{\circ}$ visual field; $.5,3,10$, and $20 \mathrm{sec}$ for the $11.4^{\circ} \times 11.4^{\circ}$ visual field; and $.5,3,10$, and $20 \mathrm{sec}$ for the nonrestricted visual field.

Each session consisted of a study phase and a test phase. In the study phase, one of the five visual field sizes was selected. The subject was successively shown 80 pictures with that visual field size at various exposure durations in a predetermined order. At each exposure duration, 20 pictures were presented. No picture was used twice for the same subject. The subject's task was merely to observe the picture for the exposure duration. While the subject was observing the picture, his eye movements were recorded on magnetic tape.

In the test phase, the subject was presented 160 pictures in succession with a fixed exposure duration of $2 \mathrm{sec}$. No restriction was made for the visual field. These pictures were composed of the 80 pictures used in the study phase and 80 new pictures. The two sets were shuffled together by the experimenter to provide a random order of presentation. Following cach picture presentation, the subject was asked to respond "yes" if he recognized the picture as having been presented in the study phase and "no" if he did not recognize it as having been presented previously. The subject was informed in advance that the correct answer for half the pictures was "yes." Eye movements were not recorded in this phase.

Prior to the first session, the subject's right-eye position was carefully adjusted. This calibration procedure required about $1 / 2 \mathrm{~h}$. When the subject was ready, the experimenter selected a visual field size and an exposure duration and signaled the subject with a buzzer to start the experiment. The subject then pressed a start button which immediately initiated appearance of the visual field on the TV screen and activated the timer. The picture was displayed for the preselected exposure duration, following which the screen went blank. The experimenter changed to the next picture and selected another exposure duration, when necessary, and signaled the subject for the next observation. The same procedure continued until all 80 stimuli were exposed.

The test phase followed the study phase after a break of a few minutes. An entire session lasted about $40 \mathrm{~min}$. After a break of $30 \mathrm{~min}$, the next session started with a new visual field size. Each subject participated in 10 sessions (each visual field size was used twice in all), requiring approximately $12 \mathrm{~h}$ altogether, divided between 2 days. On the 1st day, three subjects started with the $3.3^{\circ} \times 3.3^{\circ}$ visual field, proceeding to the no-restriction condition, and the other two subjects were given the reverse order. Two or 3 days later the subjects resumed the experiment in the respective reversed orders of presentation. 

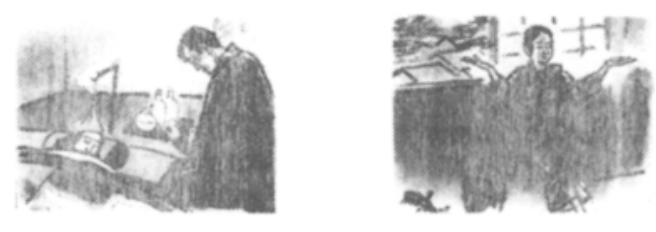

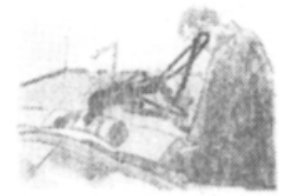

(a)

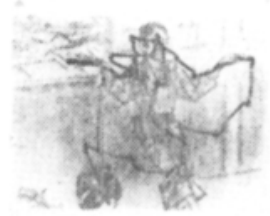

(b)
Figure 2. Two examples of pattern stimuli and eye-movement traces when a subject viewed the pictures with nonrestricted field (a) and field restricted to $3.3^{\circ} \times 3.3^{\circ}(\mathrm{b})$.

\section{Results}

Two examples of eye-movement records are shown in Figure 2 (bottom) together with the corresponding pictures (top). Figure $2 \mathrm{a}$ shows an eye-movement 10 -sec record for the stmulus picture without any restriction. Figure $2 \mathrm{~b}$ shows a $10-\mathrm{sec}$ record for the same subject with a restricted visual field of $3.3^{\circ} \times$ $3.3^{\circ}$. These records indicate some of the general characteristics of scanning. With the visual field not restricted, specific portions in the picture were repeatedly fixated without covering large portions of the picture with the fovea, implying the use of peripheral vision. On the other hand, with the visual field restricted to $3.3^{\circ} \times 3.3^{\circ}$, the picture was scanned more or less uniformly and saccadic size became smaller.

To assess the influence of narrowing the visual field, we obtained the probability of a "hit," which " was the probability of the subject's saying "yes" to pictures presented during the study phase. We also obtained the probability of a "false alarm," which was the probability of incorrectly responding "yes." Figure 3 is a plot of the percent of correct "yes" answers for each visual field, averaged across subjects as a function of the exposure duration on a logarithmic scale. The percent of "false alarms" for each visual field size averaged over all exposure durations is shown at the lower left along the ordinate. The data points for correct "yes" answers were based on 200 responses ( 5 subjects by 20 stimuli by 2 sessions) and were fitted by probit regression lines (Finney, 1971). Chi-square values for goodness of fit were $.10, .21, .80, .72$, and .72 for $3.3^{\circ} \times 3.3^{\circ}, 4.6^{\circ} \times$ $4.6^{\circ}, 7.3^{\circ} \times 7.3^{\circ}, 11.4^{\circ} \times 11.4^{\circ}$, and nonrestricted visual fields, respectively (for all values, $\mathrm{df}=2$ ). These chi-square values indicate that the goodness of fit of these lines is almost perfect because these values are well within the limits of random variation, i.e., 2.0 in this case. Lines from the two largest fieldsize conditions coincided precisely, and only one line is shown in Figure 3.
The longer the picture was displayed, the more accurate the recognition of it became. However, the locations of the functions along the duration dimension differed significantly depending upon field size. Particularly, the function for field size $3.3^{\circ} \times 3.3^{\circ}$ was shifted to the right to a great extent, indicating a serious deterioration in recognition, probably corresponding to a decrease, in the ability to extract relevant features or to construct a representation of the picture. In general, the degree of deterioration decreased as the visual field size increased. There was no further improvement, however, after $11.4^{\circ} \times$ $11.4^{\circ}$, indicating this size to be equivalent to that for normal visual perception.

To see the extent of the deterioration due to visual field reduction, an arbitrary percent correct may be chosen, from which the exposure times may be read from Figure 3. These exposure times may then be designated as the time necessary for a certain degree of recognition with corresponding visual field sizes. We chose the $70 \%$ level, as mentioned before, to calculate the recognition times as well as the standard errors by using a probit analysis. Reciprocals of the recognition times reflect the speed of processing the pictures when subjects observed them at the time of the study phase. These speeds are plotted in Figure 4 as a function of visual field size in visual angle (top) and in percent of the area of the whole pattern (bottom), both on logarithmic scales. The data from the no-restriction condition are plotted at the $100 \%$ point on the abscissa.

It appears that the speed-of-information processing increases linearly relative to logarithmic increases in visual field size, as seen in Figure 4. At a larger visual field size, processing speed reaches an upper limit and becomes constant. Thus, the relation can be

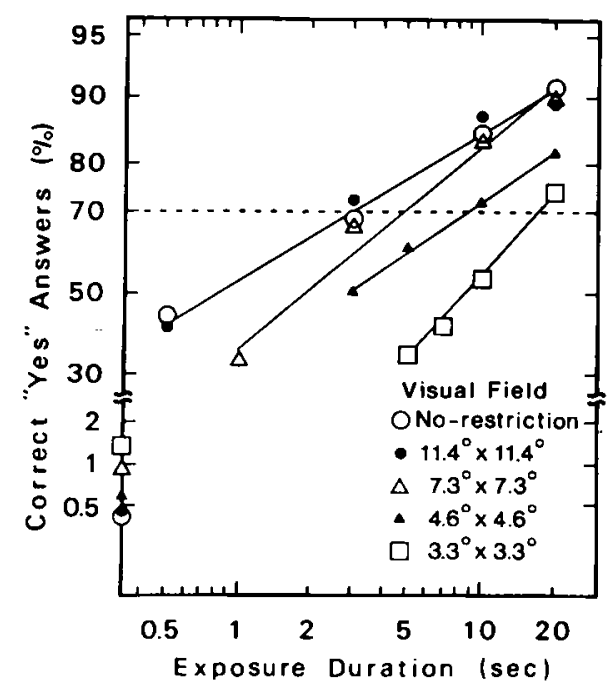

Figure 3. Percent correct "yes"' answers for pattern recognituon as a function of exposure time in the study phase of the large picture experiment. 


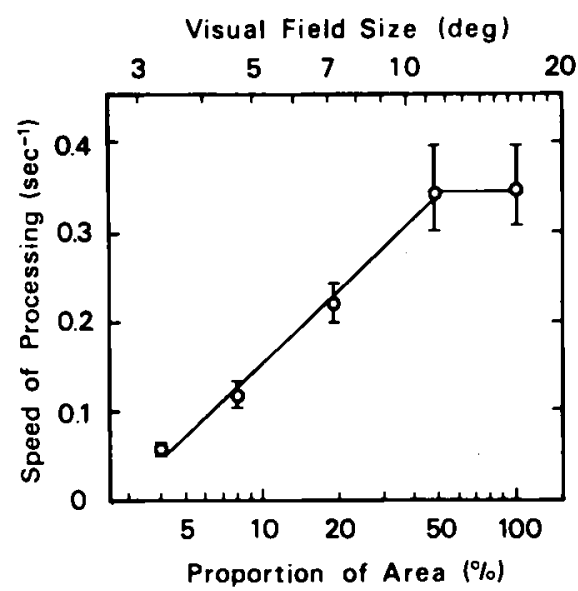

Figure 4. Speed of processing pictures as a function of visual field size in the large-picture experiment. (Bottom abscissa is percentage restricted visual field size was of entire picture size. Vertical lines indicate standard errors.)

approximated with two straight lines. The visual field size at the intersection point of the two lines may be termed the critical visual field size for pattern perception beyond which no increase in the information about the pattern takes place. This field size is the useful visual field for pattern perception when the patterns are such as those in Figures $2 \mathrm{a}$ and $2 \mathrm{~b}$. The useful visual field size was about $11.5^{\circ}$ of visual angle, or about $50 \%$ of the area of the whole pattern.

To analyze the eye-movement behavior of the subjects in the study phase, a frequency distribution of the saccadic sizes was obtained for each visual field size by utilizing the records of all study phases with this size. The results are shown in Figure 5 . In each panel, except in the case of no restriction, the visual field size is indicated by an arrow along the abscissa. The saccadic movements to the left of those arrows, or within the shaded portion, then provided the overlap between visual fields surrounding successive points. For the no-restriction case, no such overlap could be indicated. However, as we now know that the visual field beyond the useful visual field size is not necessary for pattern perception, the useful visual field size can be plotted along the abscissa of the frequency distribution curve, as in the other cases. Figure 5 indicates that when the visual field is larger than $11.5^{\circ}$ of visual angle, it moves across the picture making overlaps most of the time. When the field is made smaller, such overlapping decreases. Although overlap decreases as the field is made smaller, as much as $75 \%$ of all of the eye movements overlap immediately successive visual fields, even with the size of $3.3^{\circ} \times 3.3^{\circ}$. It appears, then, that the overlap of successive visual fields on the picture is important to gain good perception of the pattern.

\section{SMALL-PICTURE EXPERIMENT}

\section{Procedure}

All pictures employed in this experiment were of the smaller size, $10.2^{\circ} \times 13.3^{\circ}$. This size was obtained by reducing the pattern magnification (not by cutting the borders). The experimental procedure was the same as in the large-picture experiment except for the visual field size and the exposure duration. Four different visual field sizes were employed: $3.3^{\circ} \times 3.3^{\circ}, 5.2^{\circ} \times 5.2^{\circ}$, $8.1^{\circ} \times 8.1^{\circ}$, and $11.4^{\circ} \times 11.4^{\circ}$, corresponding, respectively, to $8 \%, 19 \%, 48 \%$, and $95 \%$ of the area of the entire pattern. The useful visual field size was $11.5^{\circ} \times 11.5^{\circ}$, or $50 \%$ of the entire picture according to the large-picture experiment. As we were interested in knowing whether the useful visual field size was determined by absolute size or by the size relative to the entire picture, we employed both the $11.4^{\circ} \times 11.4^{\circ}$ visual field and the $8.1^{\circ} \times 8.1^{\circ}$ visual field.

For each visual field size, four exposure durations were employed: $3,5,7$, and $10 \mathrm{sec}$ for the $3.3^{\circ} \times 3.3^{\circ}$ visual field; $1,3,5$, and $10 \mathrm{sec}$ for the $5.2^{\circ} \times 5.2^{\circ}$ visual field; $.5,1,3$, and $10 \mathrm{sec}$ for the $8.1^{\circ} \times 8.1^{\circ}$ visual field; and $.5,1,3$, and $10 \mathrm{sec}$ for the $11.4^{\circ} \times 11.4^{\circ}$ visual field. These different sets of durations were used because a slight ceiling effect was observed at the 20-sec exposure duration for the three largest field sizes in the large-picture experiment (Figure 3).

Two subjects started with the $3.3^{\circ} \times 3.3^{\circ}$ visual field size, proceeding to the $11.4^{\circ} \times 11.4^{\circ}$ visual field size on the 1 st day. The other two subjects proceeded in the reverse order. On the next day, the presentation orders were reversed.

\section{Results}

Figure 6 is a plot of percent correct "yes" answers as a function of the exposure duration on a logarithmic scale. The percent of "false alarms" for each

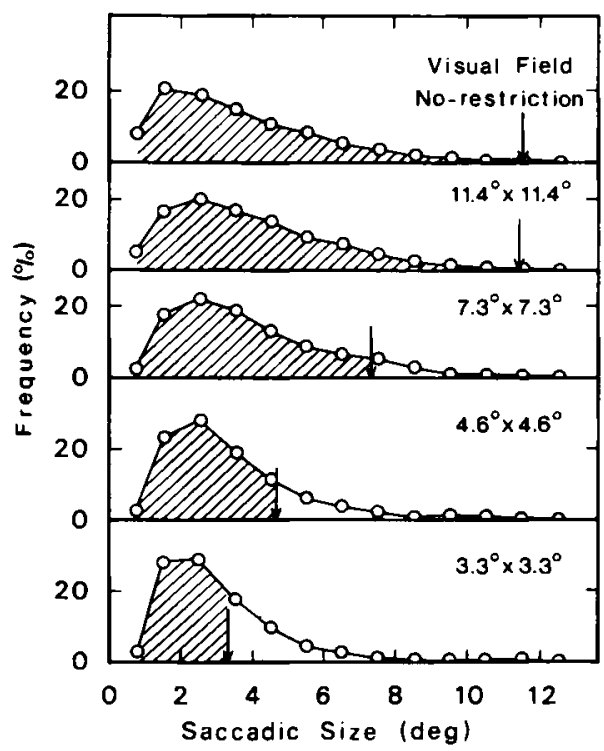

Figure 5. Distribution of saccadic eye-movement sizes for various visual field sizes in the lange-picture experiment. (In the top panel, arrow indicates useful visual field size as obtained in Figure 4. In the other panels, arrow indicates respective visual field size.) 


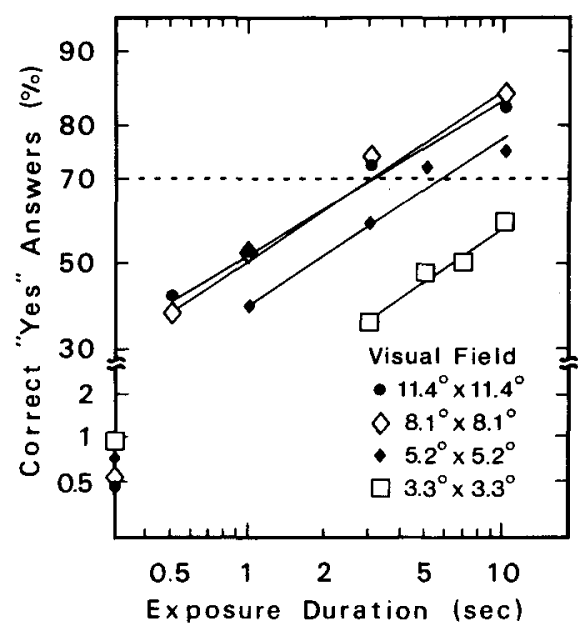

Figure 6. Percent correct "yes" answers for pattern recognition as a function of exposure time in the study phase of the small picture experiment.

visual field size is also shown at the lower left along the ordinate. Probit regression lines were fitted to these data points which were based on 160 responses ( 4 subjects by 20 stimuli by 2 sessions). The chi-square values were $.13, .52, .32$, and .40 , respectively, for the $3.3^{\circ} \times 3.3^{\circ}, 5.2^{\circ} \times 5.2^{\circ}, 8.1^{\circ} \times 8.1^{\circ}$, and $11.4^{\circ} \times 11.4^{\circ}$ visual field sizes ( $\mathrm{df}=2$ in all cases) and were well within the limits of random variation. The probit regression lines for the $8.1^{\circ} \times 8.1^{\circ}$ and $11.4^{\circ} \times 11.4^{\circ}$ field sizes were almost the same. Other properties were very similar to those in Figure 3.

To assess the influence of restricting the visual field on visual information processing, the recognition time at $70 \%$ correct was calculated as before for each visual field with the help of probit analysis. The reciprocals of these values, namely the speeds of processing the pictures, are plotted against the visual field size in Figure 7. The results were approximated again by two straight lines, and the useful visual field size can be determined from the intersection of these two lines, which is at about $8^{\circ}$ of visual angle. This value is smaller than the previous value obtained from the large-picture experiment. However, if we take the proportion of the field area to the area of the entire picture, it is about $50 \%$, which is the same as in the previous case. The determining factor for the useful visual field size is not the size itself, but the proportion of the whole pattern displayed.

To assess eye-movement behavior, the frequency distributions of saccadic sizes were obtained as before and are plotted in Figure 8. Each arrow on the abscissa indicates the size of the respective visual field. The figure indicates that when the visual field is at least $8^{\circ}$ of visual angle, or the useful visual field size, the field moves over the picture by making an overlap most of the time. When the visual field is reduced below $8^{\circ}$, the amount of overlap of the fields surrounding successive fixation points decreases. It appears that such overlap is important to gain good perception of the pattern, as pointed out before.

\section{DISCUSSION}

The present experiments suggest that the effective factor determining useful visual field size is not the absolute visual angle, but rather the percentage of the picture area $(50 \%$ in the present case). This implies that the size of the useful visual field is not only determined by peripheral characteristics, such as anatomical retinal structure or visual acuity, but also by more central characteristics such as the information-

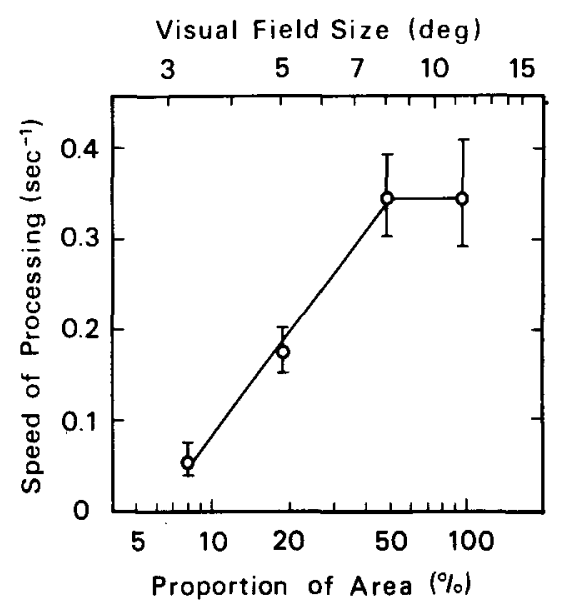

Figure 7. Speed of processing pictures as a function of visual field size in the small-picture experiment. (Bottom abscissa is percentage restricted visual field size was of entire picture size. Vertical lines indicate standard errors.)

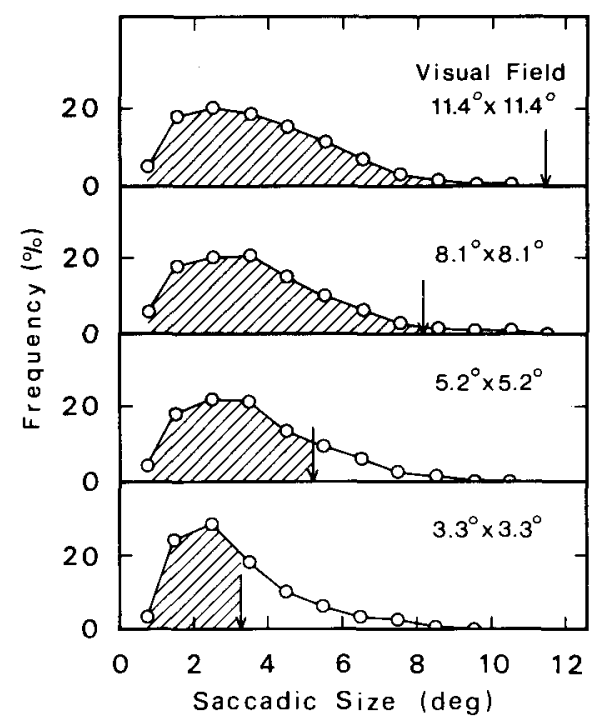

Figure 8. Distribution of saccadic eye-movement sizes for various visual field sizes in the small-picture experiment. (Arrow indicates respective visual field size.) 
processing capacity of the brain. We may suppose that, as the pattern becomes more compact, the brain reduces the useful visual field to a size such that the input which must be processed at each fixation point remains relatively constant.

When the restricted visual field size was larger than the useful visual field size, the majority of the saccades in scanning a picture were shorter than the half-size of the useful visual field in both experiments (see Figures 5 and 8). This consideration of the saccadic size data suggests two possibilities. First, a decision about where to fixate next is made before the saccade occurs, perhaps by processing the visual information in the periphery. This interpretation is supported by the data of Yarbus' experiment (1967), showing that different fixation densities for the same picture can be obtained from the same subject under different instructions. Since the useful visual field size is much wider than the foveal region, information in the visual periphery out to an eccentricity of nearly $6^{\circ}$ may be useful for information processing, as shown in the large-picture experiment.

Second, in scanning a picture, the visual system seems to operate in such a manner that the subject's useful visual field, through which visual information is taken into the visual system, scans the picture heavily overlapping itself at successive fixation points. This overlap seems to be used in integrating successive glimpses into a meaningful mental representation of the pattern. Such a conclusion was suggested by Gibson (1966), Hochberg (1968), and Gould (Note 1).

It is easily understood why Andreeva et al. (1972) and Watanabe (1971) came to the conclusion that when the visual field was restricted to $3^{\circ}$ of visual angle a marked deterioration of pattern recognition occurred. In the experiment of Andreeva et al., $3^{\circ}$ of visual angle corresponded to $2 \%$ to $10 \%$ of the area of the whole pattern, and in Watanabe's experiment, $3^{\circ}$ of visual angle corresponded to $3 \%$ of the area of the whole pattern. If the line is extrapolated to $2^{\circ}$ in Figure 4, the speed of processing will approach zero.

The speed of information processing might be investigated by measuring the time duration needed to arrive at a certain level of perception of a picture, as was done by the present authors (Ikeda \& Saida, 1978) for the case of reading sentences. However, such a subjective criterion is not reliable in the case of picture stimuli. It may vary greatly among subjects and may even fluctuate considerably in one subject. Instead, in the present investigation, we combined a study phase and a test phase to measure the accuracy of pattern perception. A question may arise whether memory decays too rapidly to use recognition as a measure of the accuracy of pattern perception. Similarly, perhaps memory capacity is too limited to hold the information of so many pictures $(80)$ as were used in the study phase. The work of various authors has answered those questions in favor of our interpretation. For example, Shepard's (1967) and Standing, Conezio, and Haber's (1970) findings showed a long decay period. Similarly, Loftus (1972) and Potter and Levy (1969) showed that great capacity exists for visual information. Finally, relatively small standard errors in Figures 4 and 7 seem to confirm that the present method is appropriate for measuring the degree of perception of picture stimuli.

\section{REFERENCE NOTE}

1. Gould, J. D. Looking at pictures. (Research Report RC4991). New York, IBM Thomas J. Watson Research Center, 1974.

\section{REFERENCES}

Andreeva, E. A., Verghiles, N. Ju., \& Lomov, B. F. [On the function of eye movements in the process of visual perception.] Voprosy Psikhologï, 1972, 18, 11-24.

ANTES, J. R., \& EDwards, D. C. Information processing in the visual periphery. Bulletin of the Psychonomic Society, 1973, 1, 351-353.

BECKER, J. Über taktilmotorische Figurwahrnehmung. Psychologische Forschung, 1935, 20, 102-158.

Chaikin, J. D., Corbin, H. H., \& Volkmann, J. Mapping a field of short-time visual search. Science, 1962, 138, 1327-1328.

Edwards, D. C., \& Goolkasian, P. A. Peripheral vision location and kinds of complex processing. Journal of Experimental Psychology, 1974, 102, 244-249.

ENGEL, F. L. Visual conspicuity, directed attention and retinal locus. Vision Research, 1971, 11, 563-576.

Finney, D. J. Probit analysis. Cambridge: Cambridge University Press, 1971.

GiBson, J. J. The senses considered as perceptual systems. Boston: Houghton-Mifflin, 1966.

HochberG, J. In the mind's eye. In R. H. Haber (Ed.), Contemporary theory and research in visual perception. New York: Holt, Rinehart and Winston, 1968.

IKEDA, M., \& SAIDA, S. Span of recognition in reading. Vision Research, 1978, 18, 83-88.

IKedA, M., SAIDA, S., \& SugiYama, T. Visual field size necessary for length comparison. Perception \& Psychophysics, $1977,22,165-170$.

IKEDA, M., \& TAKEUCHI, T. Influence of foveal load on the functional visual field. Perception \& Psychophysics, 1975, 18, 255-260.

LATour, P. L. Visual threshold during eye movements. Vision Research, 1962, 2, 261-262.

LoFTus, G. R. Eye fixations and recognition memory for pictures. Cognitive Psychology, 1972, 3, 525-551.

McConkie, G. W., \& Rayner, K. The span of the effective stimulus during a fixation in reading. Perception \& Psychophysics, $1975,17,578-586$.

Potter, M. C., \& Levy, E. I. Recognition memory for a rapid sequence of pictures. Journal of Experimental Psychology, 1969, 81, 10-15.

SANDERs, A. F. Some aspects of the selective process in the functional visual field. Ergonomics, 1970, 13, 101-117.

SHEPARD, R. N. Recognition memory for words, sentences, and pictures. Journal of Verbal Learning and Verbal Behavior, 1967, 6, 156-163.

Standing, L., Conezio, J., \& Haber, R. N. Perception and memory for pictures: Single-trial learning 2500 visual stimuli. Psychonomic Science, 1970, 19, 73-74. 
VolkmanN, F. C. Vision during voluntary saccadic eye movements. Journal of the Optical Society of America, 1962, 52, 571-578.

Volkmann, F. C., Schick, A. M. L., \& Riggs, L. A. Time course of visual inhibition during voluntary saccades. Journal of the Optical Society of America, 1968, 58, 562-569.

Watanabe, A. [Fixation points and the eye movements.] Oyo Butsuri, 1971, 40, 330-334.

YAmANE, K. [Tactile pattern perception.] Japanese Journal of $P_{s y}$ chology, 1935, 10, 327-390.
YARBus, A. L. In L. A. Riggs \& B. Haigh (Eds. and trans.), Eye movements and vision. New York: Plenum Press, 1967.

Zuber, B. L., \& STARK, L. Saccadic suppression: Elevation of visual threshold associated with saccadic eye movements. Experimental Neurology, 1966, 16, 65-79.

(Received for publication January 12, 1978; revision accepted November 28, 1978.) 